\title{
Deducing the coefficient of relationship by the amount of recombination produced during automictic parthenogenesis
}

\author{
JACO M. GREEFF* \& MARTIN H. VILLET \\ Department of Zoology and Entomology, Rhodes University, P.O. Box 94, Grahamstown, 6140, South Africa
}

\begin{abstract}
Recombination occurs in automictic parthenogens, but it is localized to the DNA between the chiasma and the telomere if central fusion (fusion of non-sister nuclei of meiosis II) occurs, and to the DNA between the chiasma and the centromere if terminal fusion (of sister nuclei of meiosis II) occurs. Independent assortment of recombined DNA leads to more variability. Here it is argued that by comparing heritabilities obtained from sib analysis of sexually-reproducing individuals with heritabilities obtained from parent-offspring regressions of automictic individuals, one can obtain an estimate of the coefficient of relationship $(r)$ between automictically produced offspring and their parents. The coefficient of relationship for different traits will differ and can be related to the average position (in relation to chiasmata) of genes affecting the trait or to the heterozygosity of the genes.
\end{abstract}

Keywords: Apis mellifera capensis, automixis, coefficient of relationship, parthenogenesis, recombination.

\section{Introduction}

A honeybee worker is normally produced by the fusion of a queen's gamete with that of a drone. Thus, in honeybees, parent-offspring regressions are normally not possible because drones and queens differ physiologically from workers. In the Cape honeybee (Apis mellifera capensis) workers can be produced in another way: by automictic parthenogenesis with central fusion (the pronucleus and one of the second division products of the first polar nucleus fuse; Verma \& Ruttner, 1983). This ability has been used (Moritz \& Hillesheim, 1985; Moritz \& Klepsch, 1985; Hillesheim $\&$ Moritz, 1987) to avoid the physiological problems and allows the estimation of heritabilities of traits using parent-offspring regressions. An assumption made for this approach is that the coefficient of relationship $(r)$ between workers and their parthenogenetically produced worker offspring is equal to 1 . The limitations of this assumption are examined here, and the logic of the argument is used in reverse to estimate $r$ in parthenogenetic offspring from measures of heritabilities.

*Present address: Department of Zoology, University of Pretoria. Pretoria, 0002, South Africa.

\section{Effects of crossing-over}

The model of central fusion in Fig. 1 explains the rationale for estimating $r$ as equal to 1 , as it predicts that the offspring will have exactly the same genotype as their parents, i.e. they are apparently clones of their parents. However, if there is any crossing-over of heterozygous loci during meiosis $\mathrm{I}, r$ will be smaller than 1 (Fig. 2). After crossing-over, heterozygous genes between the chiasmata and the telomeres stand a 50 per cent chance of becoming homozygous. In each of the 16 bivalents of the Cape honeybee there are normally two crossovers, one per arm (Verma \& Ruttner, 1983); a total of at least 32 crossovers.

Additional recombination follows crossing-over because all of the DNA between the chiasmata and the telomeres of different chromosomes assorts independently. If one considers more than one chromosome, even more recombination will arise (Table 1). Both sources of recombination undermine the assumption that $r=1$.

It should be realized that $r$ does not have the same value for all of the genes in an organism, as it depends on where the genes affecting a trait are situated relative to the chiasmata, and on how many of these genes are 


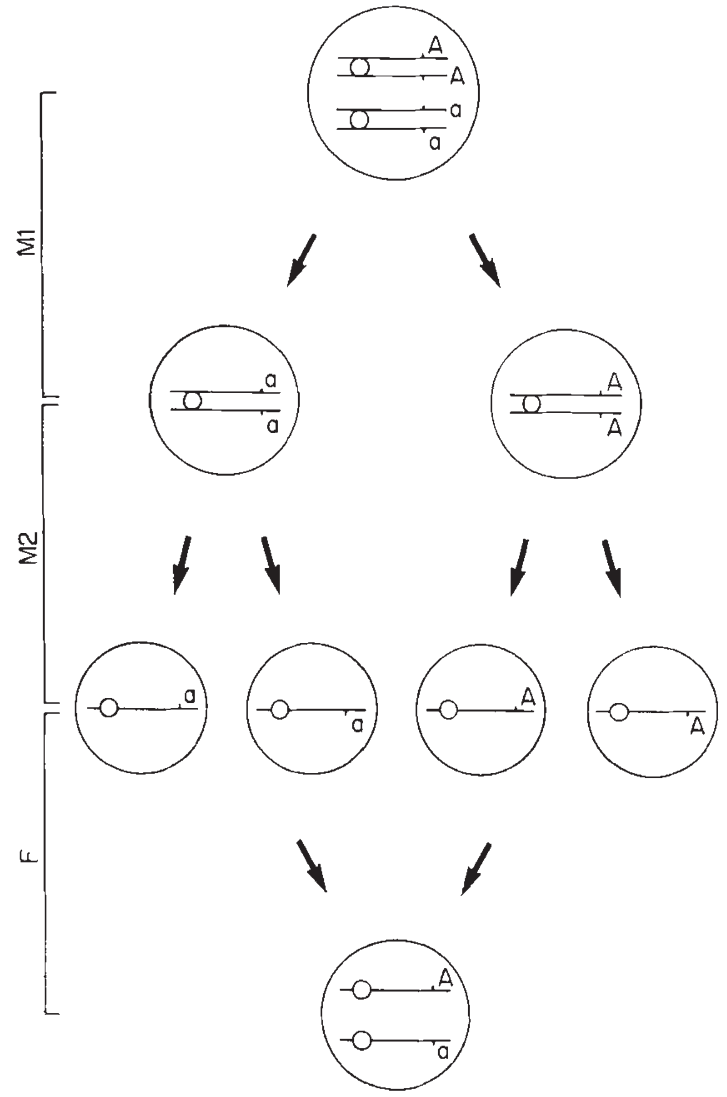

Fig. 1 Automictic parthenogenesis with central fusion, when no crossing-over occurs in meiosis I. $r=1.0 ; \mathrm{M} 1=$ meiosis I; $\mathrm{M} 2=$ meiosis $\mathrm{II} ; \mathrm{F}=$ central fusion. $A a$ is the only possible fusion product.

heterozygous. Recombinant genotypes can only be produced by a loss of heterozygosity.

Turning to the results of experiments based on the assumption that $r=1$ (Moritz \& Klepsch, 1985; Hillesheim \& Moritz, 1987), an interesting pattern shows up in 10 out of 10 estimates of heritabilities. The heritabilities obtained from parent-offspring regressions $\left(h_{\mathrm{p}}^{2}\right)$ are always smaller than heritabilities obtained by using sib analysis $\left(h_{\mathrm{s}}^{2}\right) \cdot h_{\mathrm{s}}^{2}$ consists of:

$h_{s}^{2}=\left[V_{\mathrm{A}}+0.25 V_{\mathrm{D}}+V_{\mathrm{H}}+V_{\mathrm{M}}\right] / V_{\mathrm{P}}$,

where $\quad V_{\mathrm{A}}=$ additive variance, $V_{\mathrm{D}}=$ dominance variance, $V_{\mathrm{H}}=$ variance due to common hive effects, $V_{\mathrm{M}}=$ maternal variance and $V_{\mathrm{P}}=$ phenotypic variance. $h_{\mathrm{p}}^{2}$ consists of:

$h_{\mathrm{p}}^{2}=\left[V_{\mathrm{A}}+V_{\mathrm{D}}+V_{\mathrm{M}}\right] / V_{\mathrm{p}}$.

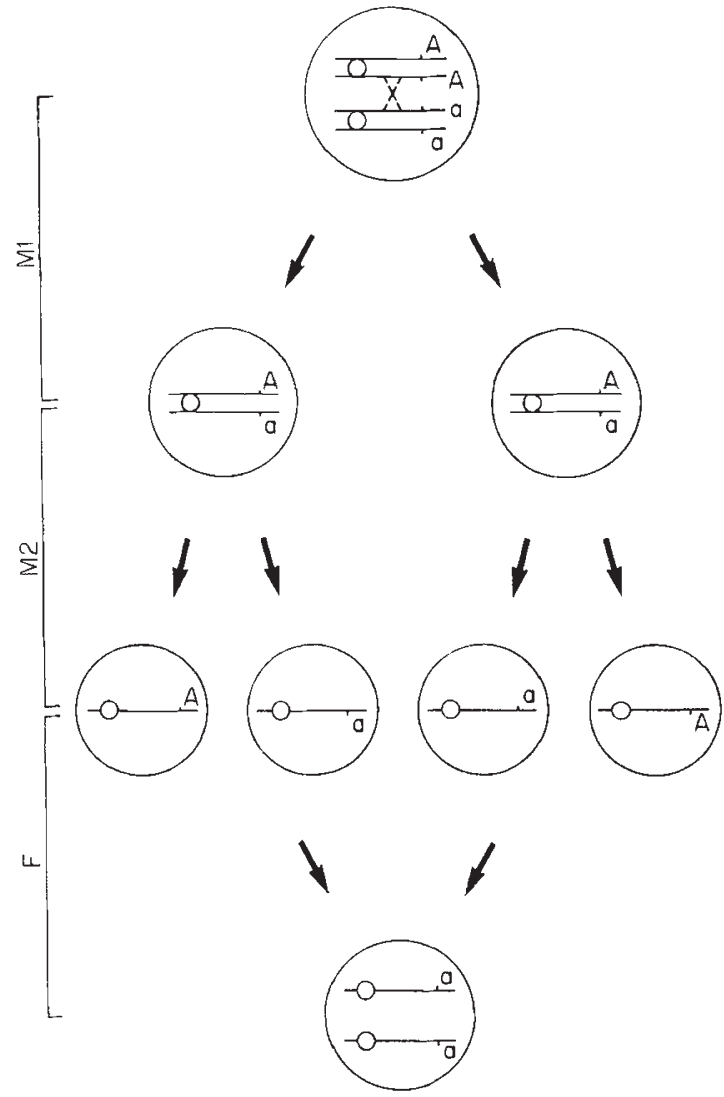

Fig. 2 Automictic parthenogenesis with central fusion, when crossing-over occurs in meiosis I. $0.5 \leq r \leq 1.0$, depending on heterozygosity and the placing of loci relative to chiasmata. $\mathrm{M} 1=$ meiosis I; $\mathrm{M} 2=$ meiosis II; $\mathrm{F}=$ central fusion. $a a$ is one of the three possible fusion products, the others being $A a$ and $A A$. The $\mathrm{F}_{1}$ frequencies of $A A: A a: a a$ are $0.25: 0.5: 0.25$.

The discrepancy between these estimates may be ascribed to variability in $h^{2}$ during different seasons of the year (Johansson \& Korkman, 1950) that affects $V_{\mathrm{H}}, V_{\mathrm{M}}$ and $V_{\mathrm{P}}$; to the additional component of a common hive environment in $h_{\mathrm{s}}^{2}$ (Hillesheim \& Moritz, 1987); and/or to an overestimate of $r$ (Moritz \& Klepsch, 1985) which will reduce the magnitude of $V_{\mathrm{A}}$ and $V_{\mathrm{D}}$ in equation 2 (cf. equations 2 and 5). An overestimate of $r$ is almost inevitable if the effects of crossing-over are ignored, and it is very likely to contribute to the above discrepancy. A difficulty is that this overestimate is not a constant and will vary according to the position (in relation to the chiasma/ta) of the genes affecting the trait and their initial heterozygosity. 
Table 1 Frequency of degrees of recombination in the offspring of an automictic parthenogenetic organism with 16 independently assorting bivalents and only one crossover per bivalent

\begin{tabular}{lc}
\hline $\begin{array}{l}\text { Number of bivalents affected } \\
\text { by crossover }\end{array}$ & $\begin{array}{l}\text { Frequency } \\
(\%)\end{array}$ \\
\hline 16 & 0.00153 \\
15 & 0.0245 \\
14 & 0.184 \\
13 & 0.86 \\
12 & 2.78 \\
11 & 6.68 \\
10 & 12.25 \\
9 & 17.50 \\
8 & 19.69 \\
7 & 17.50 \\
6 & 12.25 \\
5 & 6.68 \\
4 & 2.78 \\
3 & 0.86 \\
2 & 0.184 \\
1 & 0.0245 \\
$0^{*}$ & 0.00153 \\
\hline
\end{tabular}

${ }^{*}$ Clones, i.e. where $r=1.0$.

Thus $r$ will be different for different traits, but it can be estimated from the discrepancy between $h_{\mathrm{s}}^{2}$ and $h_{\mathrm{p}}^{2}$.

\section{Estimating $r$}

Interesting information can be obtained by reversing the whole analysis. The covariance between parent and offspring is explained as (Malecot, 1948):

$$
\operatorname{Cov}_{\mathrm{PO}}=\left[\left(\Omega+\Omega^{\prime}\right) / 2\right] V_{\mathrm{A}}+\left(\Omega \Omega^{\prime}\right) V_{\mathrm{D}}
$$

and $h_{\mathrm{p}}^{2}=\operatorname{Cov}_{\mathrm{PO}} / V_{\mathrm{P}}$,

where $\operatorname{Cov}_{\mathrm{PO}}=$ covariance of parents with offspring, $\Omega=$ average probability of an allele in an individual being present in one of the nuclei that will undergo central fusion and $\Omega^{\prime}=$ average probability of the homologous allele in that same individual being present in the other nucleus that will undergo central fusion (these definitions of $\Omega$ and $\Omega^{\prime}$ were altered from Malecot's (1948) to accommodate automictic parthenogenesis). Moritz and Klepsch (1985) set $\Omega$ equal to 1 to obtain equation 2. Because there is recombination this is clearly not the case. $\Omega$ is equal to $\Omega^{\prime}$. Setting $\Omega=\Omega^{\prime}=x$, rather than 1 , reduces equation 4 to:

$h_{\mathrm{p}}^{2}=\left[(2 x / 2) V_{\mathrm{A}}+x^{2} V_{\mathrm{D}}\right] / V_{\mathrm{P}}$
Keeping in mind that $h_{\mathrm{s}}^{2}$ of a trait can be obtained with the $V_{\mathrm{H}}$ component in equation 1 eliminated by cross-fostering workers between hives, a new mathematical manipulation becomes possible. If one determines $V_{\mathrm{D}}$ and $V_{\mathrm{A}}$ from the sib analysis and substitutes them into equation 5 along with the $h_{\mathrm{p}}^{2}$ obtained from equation 4 , it will be possible to solve for $x$. This assumes that $V_{\mathrm{A}}$ and $V_{\mathrm{D}}$ are the same for the sib analysis and the parent-offspring analysis. The term $(2 x / 2)$ in equation 5 is equal to $r$ in Falconer's (1990) equation 9.10:

$$
\operatorname{Cov}=r V_{\mathrm{A}}+u V_{\mathrm{D}} \text {. }
$$

Thus $2 x / 2=x=r$.

$r$ might be used (in a tenuous way) to make comparisons between the heterozygosity and position of genes relative to chiasmata for different traits. Unfortunately, these manipulations can lose their resolution because of two specific genetic effects. First, if a trait is controlled by more than one gene, an average value for the genes will be obtained and this, as the number of genes increases, will become more and more meaningless. Second, in honeybees, if the genes affecting a trait are linked to the sex determination gene, the trait will be more likely to be clonally propagated, because honeybees that are homozygous for the sex determination gene(s) (diploid drones) are eliminated by the workers within $6 \mathrm{~h}$ of hatching (Woyke, 1963).

These arguments have centred around the Cape honeybee but the same approach should be possible in other automictic organisms (including those that undergo terminal fusion as opposed to central fusion), as long as there is a sexually-reproducing form with which to compare. In organisms showing terminal fusion, the DNA that undergoes recombination does not lie between the chiasma and the telomere but between the chiasma and the centromere.

\section{Acknowledgements}

We acknowledge the FRD and Rhodes University for financial support and H. R. Hepburn, Ralph Kirby, Nikite Muller, Sally Ross and Costas Zachariades for critical reading of the manuscript.

\section{References}

FALCONER, D. S. 1990. Introduction to Quantitative Genetics. Longman Group (FE), Hong Kong.

HILLESHEIM, E. AND MORITZ, R. F. A. 1987. Genetic variance of physiological characters in the Cape honeybee. J. Apic. Res., 26. 30-36.

JOHANSSON, 1. AND KORKMANN, N. 1950. A study of the variation in production traits of bacon pigs. Acta Agr. Scand.. 1, $62-78$. 
malecot, G. 1948. Les Mathématiques de l'Hérédité. Masson, Paris.

MORITZ, R. F. A. AND HILLESHEIM, E. 1985. Inheritance of dominance in honeybees (Apis mellifera capensis Esch.). Behav. Ecol. Sociobiol., 17, 87-89.

MORITZ, R. F. A. AND KLEPSCH, A. 1985. Estimating heritabilities of worker characters: a new approach using laying workers of the Cape honeybee (Apis mellifera capensis Esch.) Apidologie, 156, 47-56.

VERMA, S. AND RUTTNER, F. 1983. Cytological analysis of the thelytokous parthenogenesis in the Cape honeybee (Apis mellifera capensis Esch.). Apidologie, 14, 41-57.

WOYKE, J. 1963. What happens to diploid drone larvae in a honeybee colony. J. Apic. Res., 2, 73-76. 